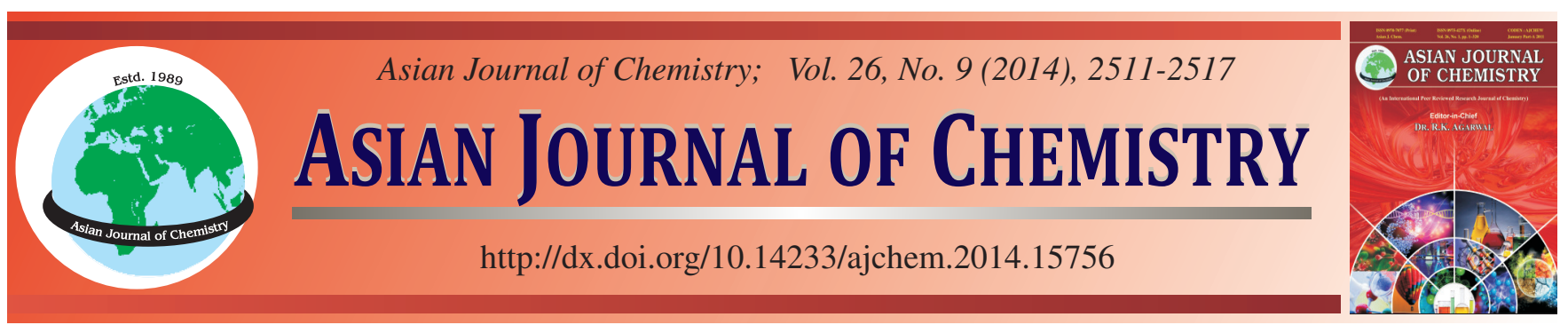

REVIEW

\title{
Chemical Kinetics Based Modelling Approaches and Tools for Investigating Complex Biological Systems
}

\begin{abstract}
P. Anbumathi ${ }^{1,2, *}$
${ }^{1}$ Department of Chemical Engineering, Indian Institute of Technology Bombay, Mumbai-400 076, India

${ }^{2}$ School of Chemical and Biotechnology, SASTRA University, Thanjavur-613 401, India

*Corresponding author: E-mail: anbumathi@scbt.sastra.edu

Biological systems are governed by their inherent complex molecular regulatory networks at multiple levels. It is the underlying chemical interactions that lead to the observed functions, phenotypic characteristics and diversity. However, it is not clear how such complex interactions at the molecular level impart specific functional characteristics of the cell/organism. This review presents a detailed account on how basic biochemical kinetics principles and computational tools are utilized to mathematically model and analyze such complex regulations. Such approaches are of crucial value in terms of (i) understanding disease mechanisms at molecular level, (ii) to predict possible drug targets (iii) to design new experiments and (iv) to combine the new experimental data along with known information to simulate drug specific pharmaco-kinetic behaviour of the system of investigation which might assist in treatment optimization. This review will also discuss a few case based examples reported in this emerging area to elucidate how such tools are successfully utilized to gain insights about biological systems for medical or drug discovery applications.

Keywords: Mathematical modelling, Kinetics, Simulation, Simulation tools, Biological systems.
\end{abstract}

\section{INTRODUCTION}

In a living cell most of the intracellular activities are due to genes and protein level chemical modifications at different instances. Chemical reactions are the working language of biological modeling which utilizes fundamental ideas of physical chemistry, kinetics, equilibrium and connect to thermodynamics. By doing so, they provide a unifying notion by which arbitrarily complex biochemical processes are expressed to gain insights about the process and dynamics either quantitatively or qualitatively ${ }^{1}$. Information and complexity are the buzz words of modern network biology. Mathematical modeling and simulation approaches can provide us with mathematically well-founded and tractable interpretations when it comes to investigating complex interaction networks ${ }^{2}$. This introductory review is an attempt to elucidate how such basic chemical kinetics based principles are utilized in quantifying biological processes with a few case based examples. This is merely an attempt to elucidate the developments in this domain in no way a complete account.

When the level of experimental and clinical data accumulates in an ever increasing rate in the past two decades with the advancements that resulted due to the successful development of high throughput experimental techniques post human genome project, it is essential to make sense of the accumulated data to better understand the biological characteristics. To gain a better understanding of the disease mechanisms and for phenotypic characterization of a disease network it is essential to combine the sparse experimental data available from several individual cell and molecular biology experiments. In such studies appropriate chemical kinetics principles are utilized to describe the underlying molecular interactions mathematically $^{3-6}$. There are several introductory reviews that guide the entire process of data collection to model development and analysis ${ }^{7-11}$. With nature's conception and publication of a dedicated open access journal, CPT: Pharmacometrics and Systems Pharmacology (http://www.nature.com/psp/index.html) since September 2012 evidently shows the momentum that this field has gained in the past decade and the amount of research that is done in this emerging research area. There are evidences where such approach has reduced the number of experiments that is essential to be performed ${ }^{11}$. The approaches towards modeling and analysis of intricate biological and disease interactions are illustrated in this review. Wide applications for such approaches are found in systematically investigating several complex biological phenomena. 
Kinetic approaches for modelling: The preliminary step to systematically model biological systems involves development of molecular interaction maps from existing experimental/ clinical data which is then transformed into appropriate mathematical formulations and later solved using appropriate computational tools. The flow chart described in Fig. 1 indicates the strategy. Various model parameters are extracted from the available experimental data through curve fitting. In a few cases where the pre-existing model parameter does not fit the model output, could indicate an existing gap in the information related to molecular interactions which could also lead to new hypothesis and experimental design. In a few cases the strategy involves repeating the above steps to get a better model which will be able to provide more insights.

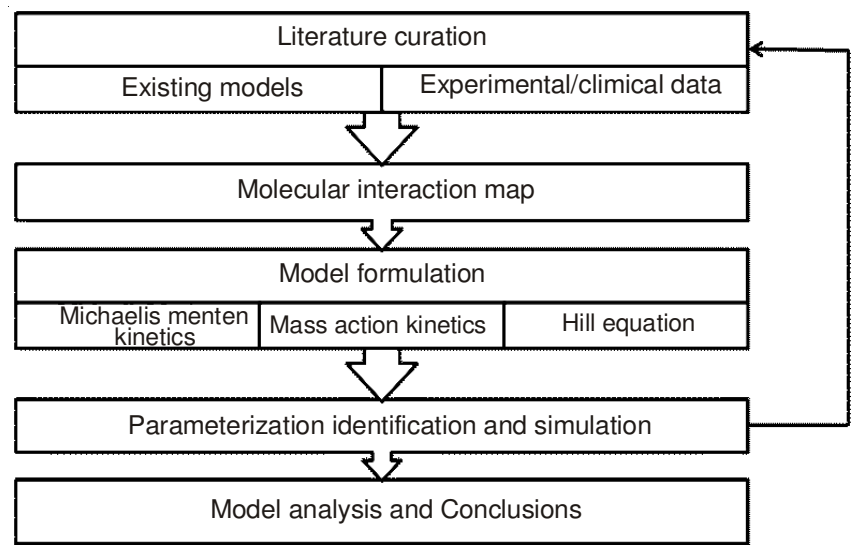

Fig. 1. Strategy followed in chemical kinetics based mathematical modeling of biological systems

Network development: Network or wiring diagram development in systems biology begins with known molecular interaction between various regulatory components deciphered from the genetics and molecular biology experiments. Similar networks in previously published models can serve as knowledge base with manual curation to a greater extend to confirm the regulatory information and to update the gaps in network. Whenever there is a lack of information/connectivity is observed a few interactions were hypothesized based on other eukaryotic model organisms which is a very commonly followed strategy in modeling and quantification of biological signal processing systems $^{8,12,13}$.

Kinetic representations: In biological systems, signal transmission occurs mostly through the following mechanisms: (i) protein-protein interactions (ii) enzymatic reactions such as protein phosphorylation dephosphorylation cycles (iii) protein degradation and (iv) through intracellular messenger proteins. Basic chemical reaction schemes such as mass action and Michaelis-Menten formulations can be used to represent all reactions ${ }^{4,5}$. A typical species that undergoes reversible protein phosphorylation dephosphorylation reaction can be depicted as:

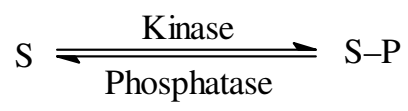

Mass action kinetics: Mass action kinetics represents an elementary kinetic principle used to describe rate of reactions based on stoichiometry and the concentrations of the reactants. For a generic reaction of the following type,

$$
\mathrm{n}_{1} \mathrm{X}_{1}+\mathrm{n}_{2} \mathrm{X}_{2}+\ldots \ldots+\mathrm{n}_{\mathrm{m}} \mathrm{X}_{\mathrm{m}} \longrightarrow \text { Products }
$$

the kinetics can be represented as

$$
-r X_{i}=k\left[X_{1}\right] n_{1}\left[X_{2}\right] n_{2} \ldots . .\left[X_{n}\right] n_{m}
$$

where ' $\mathrm{k}$ ' is the rate constant and ' $\left[\mathrm{X}_{\mathrm{i}}\right]$ ' indicate the molar concentration of the component based on cell volume. Eqn. 1 shows that the rate of chemical reaction is proportional to the product of the concentrations of the reactants.

Michaelis Menten kinetics: Michaelis Menten kinetics is commonly employed to represent rates of enzyme catalyzed reactions. This form of mathematical representation can be used for activation and inhibition of regulators and it captures the substrate saturation nonlinearity. For an enzyme catalyzed reaction of the type,

$$
\mathrm{S} \stackrel{\mathrm{E}}{\longrightarrow} \text { Product }
$$

the Michaelis Menten equation takes the form,

$$
-\mathrm{r}^{\text {Product }}=\mathrm{k}[\mathrm{E}] \frac{[\mathrm{S}]}{\left[\mathrm{K}_{0.5}\right][\mathrm{S}]}
$$

where, $[\mathrm{S}]$ and $[\mathrm{E}]$ represents the substrate and enzyme concentration, $\mathrm{k}$ and $\mathrm{K}_{0.5}$, respectively represents the Michaelis Menten rate constant and the half saturation constant of the substrate. For a continuous change in the input signal, the output signal increases and later saturates in a hyperbolic fashion (dashed line Fig. 2).

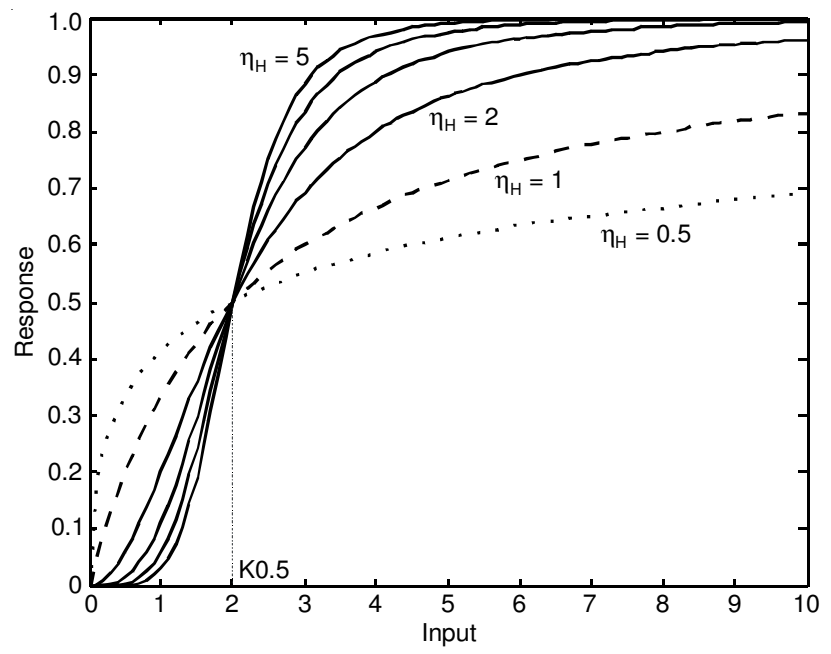

Fig. 2. Response observed for Michaelis Menten (dashed line) and Hill type (dotted and solid lines) kinetic equation formulation. Note that for increasing $\eta_{\mathrm{H}}$ the response varies from subsensitive $\left(\eta_{\mathrm{H}}=\right.$ $0.5)$, hyperbolic $\left(\eta_{\mathrm{H}}=1\right)$ to unltrasensitive $\left(\eta_{\mathrm{H}}=2,3,4\right.$ \& 5)

Hill equation: The Hill equation is used to quantify ultrasensitive or sub sensitive response which arises due to allosteric modification of proteins. It is utilized to estimate the fold change in the output/response for the given input ${ }^{4,14}$. The Hill equation is given by,

$$
\mathrm{f}=\frac{\mathrm{I}^{\eta_{\mathrm{H}}}}{\mathrm{K}_{0.5}+\mathrm{I}^{\eta_{\mathrm{H}}}}
$$

where ' $\mathrm{f}$ ' represents the fractional activation of output response with varying input 'I' and $\eta_{\mathrm{H}}$ represents Hills coefficient. The 
input signal required for $50 \%$ activation of the regulator is termed as the half saturation constant $\left(\mathrm{K}_{0.5}\right)$. Depending on the value of Hill coefficient, the shape of the stimulus response curve changes from hyperbolic to sigmoidal, indicating the measure of steepness of the curve. The Hill coefficient is computed based on the fold change in input stimulus required to take the response from $10 \%$ activation to $90 \%$ activation.

$$
\eta_{\mathrm{H}}=\frac{\log (81)}{\log \left(\frac{\mathrm{I}_{90}}{\mathrm{I}_{10}}\right)}
$$

For a response with a Hill coefficient equal to 1 , an 81 fold change in input is required to reach $90 \%$ of maximum response, which represents a typical hyperbolic Michaelis Menten response (dashed line in Fig. 2). For a Hill coefficient greater than 1 , the response tends to become sigmoidal, indicating the sensitive nature of the dose response, which is defined as ultrasensitive response (solid black line with $\eta_{H}=5$ in Fig. 2). The response curves that are less steeper than that of Michaelis Menten curve are termed as sub-sensitive responses (dotted line in Fig. 2) in which case $\eta_{H}<1$. The input-output response can be analyzed for activating a pathway/regulator by increasing the input concentration (switching on) or for deactivating a pathway by decreasing the input concentration (switching off). A system that is characterized by the same dose-response curve, irrespective of whether the dose increases or decreases is termed to be monostable. Mathematically, this implies that for a given input signal, the system has only one stable steady state that can be attained.

It is possible to observe such highly sensitive sigmoidal responses in biological systems at several instances. The classical work by Goldbeter and Khosland characterizes such sigmoidal response which arises due to reversible covalent modification. Such modification may occur due to effector/ligand biding to activate its substrate or due to phosphorylation dephosphorylation cycles ${ }^{15-17}$. Emergence of biochemical switches due to molecular level chemical interactions can be explained through Goldbeter-Koshland Equation which describes a steady-state behaviour or equilibrium characteristics for a two state biological system such as phosphorylation cycle. It is widely utilized to characterize biological systems.

Metabolic engineering: Individual reactions of large central metabolic pathways can be modeled using such elementary kinetic principles to improve cellular properties through the modification of specific biochemical reactions or the introduction of new ones, with the use of recombinant DNA technology. This is done with the notion that metabolic fluxes as determinants of cell physiology and measures of metabolic control $^{18,19}$. Application of such methods enables extension of existing pathways to obtain new chemical products, alter posttranslational protein processing and degrade recalcitrant wastes $^{18}$. There are several sub branches, which are differentially utilized for several bio-engineering studies which includes, (i) Flux balance analysis, where the flux through individual reactions are related to the total flux of the metabolic pathway and (ii) Metabolic control analysis which incorporates rate kinetics information along with flux information to precisely quantify the change in entire pathway when a single enzyme or metabolite is perturbed ${ }^{19}$.
Modelling approaches: The type of equations to be used depends on the biological questions under study. For gene and protein regulatory network interaction studies it is appropriate to use ordinary differential equations (ODEs ). In the case of ordinary differential equation modeling of cell signaling processes, it is reasonable to begin with continuum approximation and assume that the system is well mixed ${ }^{20,21}$. For example the following equation represents the general form of the ordinary differential equation for conservation of mass for any component $\mathrm{X}$ of a protein signaling based molecular interaction map,

$$
\frac{\mathrm{d}[\mathrm{x}]}{\mathrm{dt}}=\mathrm{S}_{\mathrm{x}}-\mathrm{k}_{\mathrm{d}}[\mathrm{x}] \pm\left(\mathrm{k}_{\mathrm{b}}+\mathrm{k}_{\mathrm{e}}[\mathrm{E}]\right)\left([\mathrm{x}] / \mathrm{K}_{\mathrm{m}}+[\mathrm{x}]\right) \pm \mathrm{k}_{\mathrm{m}}[\mathrm{MI}]
$$

where, $[\mathrm{x}]$ - concentration of protein ' $\mathrm{x}$ '; $\mathrm{S}_{\mathrm{x}}$ - rate of synthesis of protein ' $\mathrm{x}$ '; $\mathrm{k}_{\mathrm{d}}$ - rate constant of degradation; $\mathrm{k}_{\mathrm{b}}$ - rate of synthesis at basal level; $\mathrm{k}_{\mathrm{e}}$ - rate of synthesis in the presence of enzyme; $K_{m}$ - half saturation constant; [E] - concentration of enzyme E; MI - formation or dissociation of complex ; $\mathrm{k}_{\mathrm{m}}$ rate of complex formation or dissociation.

A set of such equation similar to equation (5) describing/ representing a complex signaling pathway or disease network can be solved using the tools listed in the later section ${ }^{22}$. Most of the mathematical models available in systems biology of cell signal processing literature adopt similar formulations to perform dynamic simulation of the systems of interest ${ }^{2,22-24}$. Such formulations can only explain or help in simulating temporal scale/ order of the processes that occur in a biological system. To simulate spatial influence partial differential equation (PDE) based formulations that accounts for the spatial dynamics along with time through a diffusion component are employed $^{25}$ and the general form of this reaction diffusion equation is given below:

$$
\frac{\partial\left[\mathrm{x}_{\mathrm{p}}\right]}{\partial \mathrm{t}}=\mathrm{D} \Delta \mathrm{x}_{\mathrm{p}}-\mathrm{v}_{\mathrm{p}}\left(\mathrm{x}_{\mathrm{p}}\right)
$$

where, $\mathrm{D}$ represents the diffusivities, $\mathrm{x}_{\mathrm{p}}$ the active form of the component any component $\mathrm{x}$ which together is assumed to form a constant pool of both the form of these components (which may not be true for a the actual system); $\Delta \mathrm{x}_{\mathrm{p}}$ represents the change in this concentration pool due to diffusion; $v_{p}$ the rate of distribution of the component in the compartment of the systems of interest.

Formulations suggested by the eqn. 6 are utilized to simulate reaction that occurs in different compartments of biological system and to describe developmental processes of biological system which are space specific such as growth pattern formation ${ }^{25-27}$. Turing for the first time demonstrated chemical influence of morphogenesis by utilizing similar formulation ${ }^{28}$.

Stochastic formulation based approaches are utilized when the details related to number of molecules and the noise of the systems of interest are investigated to study their impact on the overall systems behaviour ${ }^{6,29}$. There are models which incorporates different noise to study stochastic influence ${ }^{30}$. In most cases Gillespie algorithm based chemical master equations are formulated to simulate and analyze stochastic characteristics of the problem under investigation ${ }^{6,31-33}$. 
Model parameterization: Parameter optimization is a critical and challenging step for these types of modeling where availability of time course data is sparse, yet based on known experimental and qualitative information one can derive the parameters based on optimization techniques ${ }^{8}$. Comparing the model simulation results to experimental data is difficult because most of the experimental data is qualitative rather than quantitative $\mathrm{s}^{34}$. Different approaches utilized for parameter estimation of specific biological processes are focus for several reviews ${ }^{8,35-38}$.

Model simulation and predictions: The next step after the formulation of the model as interdependent equations and parameter identification is to study dynamical and spatiotemporal information of the system of interest through simulations. Various chemical kinetics based approaches described in the previous sections can be directly utilized to formulate the models. Models thus formed can be simulated using several numerical techniques software such as MATLAB and Mathematica. Apart from direct application of numerical techniques there are built-in tools available which can directly be employed to simulate and make unique predictions. For those with limited familiarity with solving mathematical equations there are numerous dedicated tools available to aid in solving/ simulating the problem/systems of interest. The details of which are documented in the following section. Relevant knowledge about the system is perquisite to devise testable predictions.

Modelling tools: There are several commercial and open source tools available to solve the developed models. There are also reviews which provide details on tools that are available for diverse mathematical modelling processes ${ }^{8,26}$. Table-1 below lists some of the dedicated tools and their applications with reference and web link for further reference.

Most of these tools are open source software with the user manuals available in the respective webpage to support the initial stages of exploration. Apart from these tools described there are introductory articles that clearly demonstrate how biochemical pathways are modeled mathematically using MATLAB generated ready example programs ${ }^{6}$. Similar tools and methodologies also have applications in the field of emerging field of synthetic biology where the goal based biological system developed is primarily tested with computer simulations before building/synthesizing the complete $\operatorname{organism}^{52}$.

Model analysis: Model prediction itself serves as a verification tool to check the model is able to predict and explain the existing biological data ${ }^{8,20,22}$. Specific parametric influence and the robustness of the model for parameter perturbations can be utilized to observe how the overall dynamics or phenotypic characteristics of the system under investigation get affected $^{52}$. Such quantifications are done both locally by perturbing one or two regulators and globally by systematic perturbation of the system under investigation. For oscillatory systems such as circadian rhythm and cell cycle regulation periodic sensitivities $S_{\tau}$ captures the change in the cell cycle period $\tau$ upon change in the parameter $P$ and $S_{A i}$ captures the change in the amplitude (A) or state (i) for the change in parameter $\mathrm{P}^{53}$.

$$
\mathrm{S}_{\tau}=\frac{\partial \tau}{\partial \mathrm{P}}
$$

TABLE-1

DEDICATED TOOLS AVAILABLE FOR MATHEMATICALLY MODEL AND SIMULATE BIOLOGICAL SYSTEMS

\begin{tabular}{|c|c|c|}
\hline Tools & Application & Web Link \\
\hline Gepasi & $\begin{array}{l}\text { Biochemcial kinetics simulator to model dynamical and steady state biological } \\
\text { processes, also utilized for parameter optimization }{ }^{39,40}\end{array}$ & http://www.gepasi.org/ \\
\hline Cell designer & A modelling and a structured network editing tool for biological networks ${ }^{41,42}$. & http://www.celldesigner.org/ \\
\hline Jigcell & $\begin{array}{l}\text { A sof tware modeling tool to build model and simulate biochemical regulatory } \\
\text { networks. }\end{array}$ & http://jigcell.cs.vt.edu/ \\
\hline PET & $\begin{array}{l}\text { A graphical user interface based parameter estimation toolkit to estimate rate } \\
\text { constants of molecular network models, that fit experimental data }{ }^{36} \text {. }\end{array}$ & http://mpf.biol.vt.edu/pet/ \\
\hline Simulink & A block diagram environment for multidomain simulations in MATLAB. & http://www.mathworks.in/products/simulink/ \\
\hline CompuCell3D & $\begin{array}{l}\text { An open source modeling environment and pde solver, primarly used to study } \\
\text { cellular behavior. Also used to model material and tissues in biology. }\end{array}$ & http://www.compucell3d.org/ \\
\hline E-Cell & Modeling and simulation environment for biochemical network ${ }^{43}$. & http://www.e-cell.org/ \\
\hline SBML & $\begin{array}{l}\text { Systems Biology Markup Language (SBML), a free tool for computational } \\
\text { modelling of biological processes such as metabolism and cell signaling. This } \\
\text { also supports interchangeable formats for many other tools } \mathrm{s}^{4} \text {. }\end{array}$ & http://sbml.org/Main_Page \\
\hline Flux P & Automated flux analysis tool to perform custom specific analysis ${ }^{45}$. & \\
\hline Kappa & Rule based modeling tool for biological system ${ }^{46}$. & http://www.kappalanguage.org/ \\
\hline Pajek & For large interaction networks " and graph theory based random graph. & http://pajek.imfm.si/doku.php \\
\hline Cytoscope & Open source network data integration and visualization tool ${ }^{47}$. & http://www.cytoscape.org/ \\
\hline Copasi & $\begin{array}{l}\text { COPASI is a software application for simulation and analysis of biochemical } \\
\text { networks and their dynamics }{ }^{48} \text {. }\end{array}$ & www.copasi.org/ \\
\hline Madona & Modelling and simulation tools for dynamical systems. & http://www.berkeleymadonna.com/ \\
\hline STOCHSIM & For modelling stochastic biological processes ${ }^{49}$. & \\
\hline Virtual cell & $\begin{array}{l}\text { Open source software simulation environment for Satio-temporal modeling of } \\
\text { cell signal processes }{ }^{5} \text {. }\end{array}$ & http://vcell.org/ \\
\hline Smoldyn & $\begin{array}{l}\text { A spatio stochastic simulator, utilized to perform cell-scale biochemical } \\
\text { simulations. Runs along with Virtual Cell. }\end{array}$ & http://www.smoldyn.org/ \\
\hline XPPAUT & Simulate cell signaling networks based model and numerical analysis tool. & http://www.math.pitt.edu/ bard/xpp/xpp.html \\
\hline
\end{tabular}




$$
\mathrm{S}_{\mathrm{Ai}}=\frac{\partial \mathrm{A}_{\mathrm{i}}}{\partial \mathrm{P}}
$$

where, $\mathrm{S}_{\tau}$ - Periodic sensitivity coefficient; $\mathrm{P}$ - the perturbation in parameter; $\mathrm{S}_{\mathrm{Ai}}$ - Amplitude sensitivity; $\mathrm{A}_{\mathrm{i}}$ - Measure of amplitude change and $i$ gives the state with respect to the perturbed parameter.

For systems which are not oscillatory, response to the parameter change or perturbation cab be estimated using a formulation similar to eqn. $7 \mathrm{~b}$. There are examples in literature which explicitly utilizes such methods to analyze the parameter sensitivity to characterize their robustness and rank $^{54}$.

Applications: Most of the studies reported in literature based on the modeling approaches and tools reviewed in this article have implications in identifying potential drug targets and in understanding the emergent properties of complex biochemical network interactions and network dynamics. In this section some of the crucial cases where such approaches were employed successfully for medical applications and for characterization of the complex disease networks are elucidated.

Potential drug target identification: Trypanosoma brucei is a unicellular, eukaryotic parasite which lives in the host bloodstream causes African sleeping sickness in humans and livestock. No storage mechanism for carbohydrates and central metabolic pathways such as krebs cycle or oxidative phosphorylation exist in this organism it singly depends on glycolysis for its supply of ATP. Differences between host and parasite energy metabolism are eagerly sought after as potential targets for antiparasite chemotheraphy ${ }^{55}$. Study by Bakker et $a l .{ }^{56}$ which aimed at finding the limiting step of glycolytic flux in this parasite identified potential targets for antitrypanosomal drugs through metabolic flux analysis. Their primary goal was to identify the steps that need the least inhibition to achieve a certain inhibition of the glycolytic flux, so as to avoid the inhibitor binding to the host glycolytic pathway. They found that the glucose transporter appeared to be the most promising target, followed by aldolase (ALD), glycerol-3-phosphate dehydrogenase (GDH), glyceraldehyde-3-phosphate dehydrogenase (GAPDH) and phosphoglycerate kinase. A more recent study using dynamic modeling investigates the uncertain parameter fluctuation effects due to lack precise experimental data. They also address the fragilities of the model due to the accumulation of 3-phosphoglycerate and/or pyruvate ${ }^{57}$.

Cancer: The emerging picture of molecular cell biology experiment based data and information on cancer is overwhelmingly complex which involves molecules out of many parallel signal transduction pathways which in-turn are controlled by multiple factors. The action of regulatory circuits, cross-talk between pathways and the non-linear reaction kinetics of biochemical processes complicate the understanding and prediction of the outcome of such intracellular signaling. In addition, interactions between tumor and other cell types give rise to a complex supra-cellular communication network ${ }^{58}$. They argue that if cancer is such a complex system, one aspect is to recognize, where the essence resides, i.e. to recognize cancer as a systems biology disease to provide answers to some of the pertinent questions related to cancer drug target identification and to rationalize therapies. They also show that the architecture of a signaling network is important for determining the site at which an oncologist should intervene ${ }^{58}$.

Venkatarasubramanian et al..$^{59}$ developed a tumor growth model with the hypothesis that quantifying the interactions between drugs and tumor microenvironments could be used to identify more effective anticancer strategies. Their mathematical model integrated intracellular metabolism, nutrient and drug diffusion, cell-cycle progression, cellular drug effects and drug pharmacokinetics and they assumed drug cytotoxicity to be cell-cycle phase specific and progression through the cell cycle to be dependent on ATP generation. The model consisted of a coupled set of nonlinear partial differential, ordinary differential and algebraic equations which were solved using differential-algebraic equation (DAE) solver DASPK 2.0. The therapeutic implications that emerged from such a simulation study concludes that (1) monolayer cultures are inadequate for accurately determining therapeutic effects in vitro, (2) decreasing the diffusivity of paclitaxel could increase its efficacy and (3) measuring the proliferation fraction in tumors could enhance the prediction of therapeutic efficacy. This is one such example where a combination of approaches prescribed in this review is utilized effectively.

A systematic framework was adopted to predict the sigmoidal transition of effective dose response, maximal effect $\left(\mathrm{A}_{\max }\right)$, half maximal activity $\left(\mathrm{EC}_{50}\right)$ and the concentration at which the drug response reached an absolute inhibition of $50 \%$ $\left(\mathrm{IC}_{50}\right)$ of 24 compounds on 470 cell lines was generated inview to aid the clinical trials with robust preclinical model systems. The mail goal of the study was to describe a large (encyclopedic) cell line collections based experimental and preclinical modeling study for cancer: Cancer Cell Line Encyclopedia (CCLE). Both known and novel biomarker response and sensitivity was demonstrated for several cancel cell lines collected in this database ${ }^{60}$. Other articles published in the same issue of 'Nature Outlook-Physicist take on Cancer' highlights how diverse mathematical modelling approaches have successfully been developed and utilized to predict tumor growth rate, cell migration rates, right drug target, right combination of drugs, optimal dose and sequence.

Quantification of cell signaling pathways: Towards understanding the disease mechanism several cell signaling pathways are characterized using the approaches reviewed in this section. Biological signal processing systems consists of protein - protein interactions which act together and converge to activate or inhibit a gene product which can bring in the essential response to the stimuli. The typical interactions observed in such process include direct interaction of one or more proteins, enzymatic or covalent modification, phosphorylationdephophorylation cycles and gene-protein (transcription activator/inhibitor) interaction. Detailed characterization and properties of such commonly observed components of network modules are the focus of several reviews ${ }^{3,4,61,62}$.

The negative interaction mediated regulation between the tumor suppressor p53 and the oncogene Mdm2 has been widely investigated though kinetic modeling based computational frameworks ${ }^{23,24}$. In mammalian systems under DNA damage, activation of p53 executes cell cycle arrest and initiates 
DNA repair. If irreparable when under excessive damage, the p53-mediated apoptotic pathway gets activated to bring about the cell death. Mathematical modelling demonstrated the effect of reducing the gene copy number of the p53 which might result in tumor formation by triggering proliferation of cells containing damaged DNA ${ }^{63}$. Geva-Zatorsky et al. ${ }^{64}$ along with experiments used modeling framework to elucidate the oscillatory nature of this network and to characterize the possible source of variability in oscillation as low-frequency noise in protein production rates, rather than noise in other parameters such as degradation rates. Study by Batchelor et al. ${ }^{65}$ emphasizes the importance of collecting quantitative dynamic information at high temporal resolution for understanding the regulation of signaling pathways and opens new ways to manipulate p53 pulses to ask questions about their function in response to DNA damage. Apart from the negative interaction between $\mathrm{p} 53$ and Mdm2 the upstream signaling kinases, ATM and Chk 2 and their negative interaction through Wip1 contributes in maintaining the uniform shape of $\mathrm{p} 53$ pulses. They propose that $\mathrm{p} 53$ pulses are the result of repeated initiation by ATM which is re-activated by persistent DNA damage.

Tumor necrosis factor $(\mathrm{TNF} \alpha)$ is a potent pro-inflammatory cytokine that plays an important role in immunity and inflammation, in the control of cell proliferation, differentiation and apoptosis. Simulation study by Cho et l. $^{2}$ shows qualitative validation of the interactions in comparison with experimental results for this pathway. Their approach is also applicable to further predict the signaling behaviour of NF- $\mathrm{KB}$ in a quantitative manner for any variation of the ligand, TNF $\alpha$. $\mathrm{NF}-\kappa \mathrm{B}$ is a principal transcription factor, is an exquisite example of how cellular signaling pathways can be regulated to produce different yet specific responses to different inflammatory insults. Mathematical models, tightly linked to experiment, have been instrumental in unraveling the forms of regulation in NF- $\mathrm{KB}$ signaling and their underlying molecular mechanisms ${ }^{66}$. This perspective article also provides a detailed comparative account of various models and their key contributions. Interconnectivity and the cross talk between $\mathrm{p} 53, \mathrm{TNF} \alpha$ and $\mathrm{NF}-\kappa \mathrm{B}$ pathways is the topic of interest for several mathematical modelling studies ${ }^{67-69}$. These are the key regulators which are identified to have lost their crucial controlling and regulatory decision making ability due to mutations in several cancer and tumors.

Signal transduction pathways control cellular responses to stimuli, but it is unclear how molecular information is processed as a network ${ }^{68}$. Use of mathematical models therefore not only helps in better understanding of the governing signaling networks through quantifications, it is also possible to develop prognostic strategies for several complex diseases like cancer ${ }^{70}$. The revolutionary modern biology that aims to design organisms with specific/desired properties relies on detailed chemical kinetic analysis to a greater extend before the actual synthesis ${ }^{51}$.

\section{Conclusion}

To understand the fundamental principles governing biological systems which consist of complex network interaction at multiple levels, it is essential to have interdisciplinary biochemical kinetics, mathematics, physics and computational tools based combinatorial approach. The current review elucidates this through documenting the methods adopted, approaches and tools utilized commonly and through a few specific case studies based on successful examples reported in literature. Other than these well established methods and tools there are more avenues to create accurate problem specific innovative approaches. Comparing to the huge amount of critical experiments that needs to be done to understand the disease interaction networks to identify critical drug targets mathematical model based approaches are less expensive and more effective. Complementary approach of mathematical modeling along with well designed experiments is generally prescribed for more accurate understanding.

\section{ACKNOWLEDGEMENTS}

The author acknowledged Prof. S. Bhartiya and Prof. K.V. Venkatesh, IIT Bombay for useful discussions. This work was supported by the Prof. TRR Research grant provided by SASTRA University.

\section{REFERENCES}

1. M.A. Gibson and E. Mjolsness, in eds.: J.M. Bower and H. Bolouri, Modeling the Activities of Single Genes in Computational Modeling of Genetic and Biochemical Networks, MIT Press, pp. 1-48 (2001).

2. K.-H. Cho, S.-Y. Shin, H.-W. Lee and O. Wolkenhauer, Genome Res., 13, 2413 (2003).

3. J.J. Tyson, B. Novak, G.M. Odell, K. Chen and C.D. Thron, Trends Biol. Sci., 21, 89 (1996).

4. J.E. Ferrell, Trends Biochem. Sci., 21, 460 (1996).

5. U.S. Bhalla and R. Iyengar, Science, 283, 381 (1999).

6. M. Ullah, H. Schmidt, K.-H. Cho and O. Wolkenhauer, IEE Proc. Syst. Biol., 153, 53 (2006).

7. E. Klipp, Yeast, 24, 943 (2007).

8. J.C. Sible and J.J. Tyson, Methods, 41, 238 (2007).

9. J.R. Swedlow, S.E. Lewis and I.G. Goldberg, Nat. Cell. Biol., 8, 1190 (2006).

10. K.A. Janes and M.B. Yaffe, Nat. Rev. Mol. Cell Biol., 7, 820 (2006).

11. J.B. Brown and Y. Okuno, Chem. Biol., 19, 23 (2012).

12. K.C. Chen, L. Calzone, A. Csikasz-Nagy, F.R. Cross, B. Novak and J.J. Tyson, Mol. Biol. Cell, 15, 3841 (2004).

13. A. Sveiczer, A. Csikasz-Nagy, B. Gyorffy, J.J. Tyson and B. Novak, Proc. Nat. Acad. Sci., 97, 7865 (2000).

14. P.K. Vinod and K.V. Venkatesh, J. Indian Inst. Sci., 88, 1 (2008).

15. A. Goldbeter and D.E. Koshland, Proc. Nat. Acad. Sci., 78, 6840 (1981).

16. A. Goldbeter and D.E. Koshland, J. Biol. Chem., 259, 14441 (1984).

17. A. Goldbeter, Biochemical Oscillations and Cellular Rhythms, The Molecular Bases of Periodic and Chaotic Behaviour, Cambridge University Press (1996).

18. J.E. Bailey, Science, 252, 1668 (1999).

19. G. Stephanopoulos, Metab. Engg., 1, 1 (1999).

20. J.J. Tyson, K.C. Chen and B. Novak, Nat. Rev. Mol. Cell Biol., 2, 908 (2001).

21. B.B. Aldridge, J.M. Burke, D.A. Lauffenburger and P.K. Sorger, Nat. Cell Biol., 8, 1195 (2006).

22. P. Anbumathi, S. Bhartiya and K.V. Venkatesh, Syst. Synth. Biol., 5, 115 (2011).

23. A. Ciliberto, B. Novak and J.J. Tyson, Cell Cycle, 4, 488 (2005).

24. I. Bose and B. Ghosh, J. Biosci. Aug., 32, 991 (2007).

25. B.N. Kholodenko, Nat. Rev. Mol. Cell Biol., 7, 165 (2006).

26. B.M. Slepchenko, J.C. Schaff, J.H. Carson and L.M. Loew, Ann. Rev. Biophys. Biomol. Struct., 31, 423 (2002).

27. A. Csikasz-Nagy, B. Gyorffy, W.G. Alt, J.J Tyson and B. Novak, Yeast, 25, 59 (2008).

28. A.M. Turing, Biol. Sci., 237, 37 (1952).

29. D. Fange and J Elf, PLOS Comput. Biol., 2, e80 (2006).

30. R. Steuer, J. Theor. Biol., 228, 293 (2004).

31. D.T. Gillespie, J. Comput. Phys., 22, 403 (1976). 
32. D.T. Gillespie, J. Phys. Chem., 81, 2340 (1977).

33. D.T. Gillespie, Ann. Rev. Phys. Chem., 58, 35 (2007).

34. T.D. Panning, L.T. Watson, N.A. Alle, K.C. Chen, C.A. Shaffer and J.J. Tyson, J. Global Optimization, 40, 719 (2008).

35. C.T.H. Baker, G.A. Bocharova, J.M. Fordd, P.M. Lumbb, S.J. Nortonb, C.A.H. Paula, T. Junte, P. Krebse and B. Ludewige, J. Comp. Appl. Math., 184, 50 (2005).

36. J.W. Zwolak, J.J. Tyson and L.T. Watson, J. Comp. Biol., 12, 48 (2005).

37. M. Quach, N. Brunel and F. dAlche-Buc, Bioinformatics, 23, 3209 (2007).

38. D. Yu and F. Liu, Chaos, 18, 043108 (2008).

39. P. Mendes, Comput. Appl. Biosci., 9, 563 (1993)

40. P. Mendes, Trends Biochem. Sci., 22, 361 (1997).

41. A. Funahashi, M. Morohashi, H. Kitano and N. Tanimura, Biosilico, 1, 159 (2003).

42. A. Funahashi, Y. Matsuoka, A. Jouraku, M. Morohashi, N. Kikuchi and H. Kitano, Proc. IEEE, 96, 1254 (2008).

43. M. Tomita, K. Hashimoto, K. Takahashi, T.S. Shimizu, Y. Matsuzaki, F. Miyoshi, K. Saito, S. Tanida, K. Yugi, J.C. Venter and C.A. Hutchison III, Bioinformatics, 15, 72 (1999).

44. M. Hucka, A. Finney, H.M. Sauro, H. Bolouri, J.C. Doyle, H. Kitano, A.P. Arkin, B.J. Bornstein, D. Bray, A. Cornish-Bowden, A.A. Cuellar, S. Dronov, E.D. Gilles, M. Ginkel, V. Gor, I.I. Goryanin, W.J. Hedley, T.C. Hodgman, J.-H. Hofmeyr, P.J. Hunter, N.S. Juty, J.L. Kasberger, A. Kremling, U. Kummer, N. Le Novere, L.M. Loew, D. Lucio, P. Mendes, E. Minch, E.D. Mjolsness, Y. Nakayama, M.R. Nelson, P.F. Nielsen, T. Sakurada, J.C. Schaff, B.E. Shapiro, T.S. Shimizu, H.D. Spence, J. Stelling, K. Takahashi, M. Tomita, J. Wagner, J. Wang and the rest of the SBML Forum, Bioinformatics, 19, 524 (2003).

45. B.E. Ebert, A.-L. Lamprecht, B. Steffen and L.M. Blank, Metabolites, 2, 872 (2012).

46. V. Danos, J. Feret, W. Fontana, R. Harmer and J. Krivine, in eds.: L. Caires and V.T. Vasconcelos, Rule-Based Modelling, Symmetries, Refinements, CONCUR 2007, LNCS 4703, Springer-Verlag Berlin Heidelberg, pp. 17-41 (2007).

47. P. Shannon, A. Markiel, O. Ozier, N.S. Baliga, J.T. Wang, D. Ramage, N. Amin, B. Schwikowski and T. Ideker, Genome Res., 13, 2498 (2003).

48. S. Hoops, S. Sahle, R. Gauges, C. Lee, J. Pahle, N. Simus, M. Singhal, L. Xu, P. Mendes and U. Kummer, Bioinformatics, 22, 3067 (2006).

49. N. LeNovere and T.S. Shimizu, Bioinformat. Appl. Note, 17, 575 (2001).

50. L. Loew and J. Schaff, Trends Biotechnol, 19, 401(2001).

51. A.C. Foster and G.M. Church, Mol. Syst. Biol., 2, 45 (2006).

52. S. Bhartiya, N. Chaudhary, K.V. Venkatesh and F.J. Doyle, J. R. Soc. Interface, 3, 383 (2006).
53. J. Stelling, E.D. Gilles and F.J. Doyle, Proc. Natl. Acad. Sci., 101, $13210(2004)$

54. J.H. Parmar, S. Bhartiya and K.V. Venkatesh, Mol. Biosyst., 7, 1138 (2011).

55. C.E. Clayton and P. Michels, Parasitol. Today, 12, 465 (1996)

56. B.M. Bakker, P.A.M. Michelsi, F.R. Opperdoesi and H.V. Westerhoff, J. Biol. Chem., 274, 14551 (1999).

57. F. Achcar, E.J. Kerkhoven, B.M. Bakker, M.P. Barrett and R. Breitling, PLOS Comput. Biol., 8, e1002352 (2012).

58. J.J. Hornberg, F.J. Bruggeman, H.V. Westerhoff and J. Lankelma, BioSystems, 83, 81 (2006).

59. R. Venkatasubramanian, M.A. Henson and N.S. Forbes, J. Theor. Biol., 253, 98 (2008)

60. J. Barretina, G. Caponigro, N. Stransky, K. Venkatesan, A.A. Margolin, S. Kim, C.J. Wilson, J. Lehár, G.V. Kryukov, D. Sonkin, A. Reddy, M. Liu, L. Murray, M.F. Berger, J.E. Monahan, P. Morais, J. Meltzer, A. Korejwa, J. Jané-Valbuena, F.A. Mapa, J. Thibault, E. Bric-Furlong, P. Raman, A. Shipway, I.H. Engels, J. Cheng, G.K. Yu, J. Yu, P. Aspesi Jr, M. de Silva, K. Jagtap, M.D. Jones, L. Wang, C. Hatton, E. Palescandolo, S. Gupta, S. Mahan, C. Sougnez, R.C. Onofrio, T. Liefeld, L. MacConaill, W. Winckler, M. Reich, N. Li, J.P. Mesirov, S.B. Gabriel, G. Getz, K. Ardlie, V Chan, V.E. Myer, B.L. Weber, J. Porter, M. Warmuth, P. Finan, J.L. Harris, M. Meyerson, T.R. Golub, M.P. Morrissey, W.R. Sellers, R. Schlegel and L.A. Garraway, Nature, 483, 603 (2012).

61. B. Novak and J.J. Tyson, Nat. Rev. Mol. Cell Biol., 9, 981 (2008).

62. J.E. Ferrell Jr., Y.-C. Tsai and Q. Yang, Cell, 144, 874 (2011).

63. B. Ghosh and I. Bose, Phys. Biol., 3, 29 (2006).

64. N. Geva-Zatorsky, N. Rosenfeld, S. Itzkovitz, R. Milo, A. Sigal, E. Dekel, T. Yarnitzky, Y. Liron, P. Polak, G. Lahav and U. Alon, Mol. Syst. Biol., Article number: 2006.0033 (2006).

65. E. Batchelor, C.S. Mock, I. Bhan, A. Loewer and G. Lahav, Molecul. Cell, 30, 277 (2008).

66. R. Cheong, A. Hoffmann and A. Levchenko, Mol. Syst. Biol., 4, 192 (2008).

67. T. Lipniacki, R. Bertolusso and K. Puszynski, IET Syst. Biol., 3, 356 (2009).

68. K.A. Janes, J.G. Albeck, S. Gaudet, P.K. Sorger, D.A. Lauffenburger and M.B. Yaffe, Science, 310, 1646 (2005).

69. K.A. Janes, S. Gaudet, J.G. Albeck, U.B. Nielsen, D.A. Lauffenburger and P.K. Sorger, Cell, 124, 1225 (2006).

70. H. Busch, D. Camacho-Trullio, Z. Rogon, K. Breuhahn, P. Angel, R. Eils and A. Szabowski, Mol. Syst. Biol., 4, 199 (2008). 